\title{
Intrinsic Tyrosine Fluorescence as a Tool To Study the Interaction of the Shaker B "Ball" Peptide with Anionic Membranes ${ }^{\dagger}$
}

\author{
José A. Poveda, ${ }^{\ddagger}$ Manuel Prieto, ${ }^{\S}$ José A. Encinar, ${ }^{\ddagger}$ José M. González-Ros, ${ }^{\ddagger}$ and C. Reyes Mateo ${ }^{*} \pitchfork$ \\ Instituto de Biología Molecular y Celular, Universidad Miguel Hernández, E-03202 Elche (Alicante), Spain, and Centro de \\ Química-Física Molecular, Instituto Superior Técnico, Av. Rovisco Pais, P-1049-001, Lisboa, Portugal
}

Received November 15, 2002; Revised Manuscript Received February 26, 2003

\begin{abstract}
Steady-state and time-resolved fluorescence from the single tyrosine in the inactivating peptide of the Shaker B potassium channel (ShB peptide) and in a noninactivating peptide mutant, ShB-L7E, has been used to characterize their interaction with anionic phospholipid membranes, a model target mimicking features of the inactivation site on the channel protein. Partition coefficients derived from steady-state anisotropy indicate that both peptides show a high affinity for anionic vesicles, being higher in ShB than in ShB-L7E. Moreover, differential quenching by lipophilic spin-labeled probes and fluorescence energy transfer using trans-parinaric acid as the acceptor confirm that the ShB peptide inserts deep into the membrane, while the ShB-L7E peptide remains near the membrane surface. The rotational mobility of tyrosine in membrane-embedded ShB, examined from the decay of fluorescence anisotropy, can be described by two different rotational correlation times and a residual constant value. The short correlation time corresponds to fast rotation reporting on local tyrosine mobility. The long rotational correlation time and the high residual anisotropy suggest that the ShB peptide diffuses in a viscous and anisotropic medium compatible with the aliphatic region of a lipid bilayer and support the hypothesis that the peptide inserts into it as a monomer, to configure an intramolecular $\beta$-hairpin structure. Assuming that this hairpin structure behaves like a rigid body, we have estimated its dimensions and rotational dynamics, and a model for the peptide inserted into the bilayer has been proposed.
\end{abstract}

The inactivating Shaker B $(\mathrm{ShB})^{1}$ peptide comprises the 20 N-terminal amino acids $\left(\mathrm{H}_{2} \mathrm{~N}\right.$-MAAVAGLYGLGEDRQHRKKQ) of each subunit in the Shaker B potassium channel, the so-called inactivating "ball", responsible for inducing fast, N-type inactivation in this and many other related or unrelated channels $(1-6)$. Fast inactivation implies the physical occlusion of the channel's cytoplasmic mouth (7), with the ball peptide acting as an open channel blocker. The site on the channel where the ball peptide interacts consists of a hydrophobic protein pocket, separated from the cytoplasm by a region with a negative surface potential ( 8 , 9). Such channel domains can be partly mimicked by anionic phospholipid membranes, which also contain a hydrophobic region (the hydrophobic bilayer) and a negatively charged surface. Thus, anionic phospholipid vesicles have been used as model targets to gain insight into the molecular events in which the ShB peptide might be involved during channel inactivation (10-14). Fourier transform IR spectroscopy, differential scanning calorimetry, and steady-state fluorescence

\footnotetext{
Partly supported by grants from the Portugal-Spain Cooperation HP1999-0049, BFI 2002-03410 from Spanish DGI, and PI020606 from Instituto de Salud Carlos III.

* To whom correspondence should be addressed. Phone: +34 966658469. Fax: +34 966658758. E-mail: rmateo@umh.es.

† Universidad Miguel Hernández.

$\S$ Instituto Superior Técnico.

${ }^{1}$ Abbreviations: Shaker B K ${ }^{+}$channel, one of the spliced variants of the voltage-dependent, fast-inactivating $\mathrm{K}^{+}$channels coded in the Shaker locus of Drosophila; $\mathrm{ShB}$ peptide, inactivating peptide of the Shaker B K ${ }^{+}$channel; PC, phosphatidylcholine from egg yolk; PA, phosphatidic acid prepared from egg yolk PC; $t$-PnA, trans-parinaric acid; LUV, large unilamellar vesicles; nNS, $n$-( $N$-oxy-4,4-dimethyloxazolidin-2-yl)stearic acid.
}

studies of fluorophore-labeled ShB peptide suggest that the ShB peptide (a) binds to the vesicle surface with high affinity, (b) readily adopts a strongly hydrogen-bonded intramolecular $\beta$-hairpin structure, and (c) becomes inserted into the hydrophobic bilayer in a monomeric form. In contrast, a noninactivating mutant peptide ShB-L7E also binds to the phospholipid vesicles but is unable to either form the characteristic $\beta$-structure or get into the hydrophobic lipid bilayer.

ShB peptide contains a single tyrosine residue and no tryptophan. The intrinsic fluorescence of tyrosine has been scarcely used to explore the interaction of peptides and proteins with lipid membranes, mainly because of the low fluorescence quantum yield, low extinction coefficient and short absorption wavelength, and low sensitivity to changes in the surrounding environment. Instead, most peptidemembrane interaction studies use peptides or proteins covalently labeled with extrinsic fluorophores, so that their interaction with membranes is determined indirectly from changes in the fluorescence properties of the covalently bound probe $(12,15)$. Nonetheless, despite the apparent disadvantages mentioned above, tyrosine shows a high intrinsic anisotropy and a fluorescence lifetime optimal to characterize nanosecond and subnanosecond motions in peptides and proteins (16), and therefore, it could be a useful tool to study structural and dynamic changes in peptides upon interaction with membranes. In this work we have exploited the intrinsic fluorescence of the wild-type ShB peptide, as well as that of the mutant, noninactivating ShB-L7E peptide, both having a single tyrosine residue at position 8 in their sequences, to gain further insight on the interaction and insertion of these peptides into anionic membranes. Steady- 
state and time-resolved fluorescence studies, including quenching, energy transfer, and fluorescence anisotropy experiments, have been carried out. The results confirm that the ShB peptide inserts into the anionic lipid membrane in a monomeric form to configure an intramolecular $\beta$-hairpin structure, while the ShB-L7E remains grafted to the membrane surface. Additionally, the size and rotational dynamics of the $\beta$-hairpin from ShB have been estimated from the analysis of the tyrosine time-resolved fluorescence anisotropy decay, and a structural model for the membrane-inserted peptide has been proposed.

\section{MATERIALS AND METHODS}

Sample Preparation. The wild-type ShB peptide $\left(\mathrm{H}_{2} \mathrm{~N}\right.$ MAAVAGLYGLGEDRQHRKKQ) and the noninactivating mutant peptide ShB-L7E $\left(\mathrm{H}_{2} \mathrm{~N}-\mathrm{MAAVAGEYGLGEDRQH-}\right.$ RKKQ) were synthesized and purified as described previously (12). The fluorescent probe trans-parinaric acid ( $t$-PnA) and the quencher spin-labeled probes 5-doxylstearic acid (5NS) and 16-doxylstearic acid (16NS) were purchased from Molecular Probes (Eugene, OR) and used without further purification. 5-Methoxyindole was from Aldrich Chemical Co. (Milwaukee, WI). The phospholipids phosphatidylcholine (PC) and phosphatidic acid (PA) (Avanti Polar Lipids, Birmingham, AL) were derived from egg yolk PC. All other compounds were of analytical or spectroscopic reagent grade. Doubly distilled and deionized water was used throughout this work.

Multilamellar vesicles were prepared at $1.3 \mathrm{mM}$ phospholipid concentration by resuspending the dried lipid in the required buffer (10 $\mathrm{mM}$ Hepes, $\mathrm{pH}$ 6, $100 \mathrm{mM} \mathrm{NaCl}$ ), then heating, and vortexing. Large unilamellar vesicles (LUVs) were prepared from the multilamellar suspensions by pressure extrusion through $0.1 \mu \mathrm{m}$ polycarbonate filters (Nucleopore, Cambridge, MA). The peptides were externally added to the lipid suspensions from stock solutions to a final peptide concentration of $50 \mu \mathrm{M}$.

Absorption and Fluorescence Spectroscopy. Absorption spectra were obtained in a CAMSPEC M350 spectrophotometer. Fluorescence measurements were recorded with a SLM-8000C spectrofluorometer using cuvettes with $5 \mathrm{~mm}$ path length. For all of the experiments, the sample absorbance at $275 \mathrm{~nm}$ was $<0.05$. For the anisotropy determination, the corresponding vertically and horizontally polarized emission intensities, elicited by vertically polarized excitation using Glan-Thompson polarizers, were corrected for background scattering. The lipid concentration was low enough to prevent a significant light scattering of the solution that could result in an artificial depolarization of the fluorescence.

The decay of the total fluorescence intensity and those of the parallel and perpendicular components were recorded at $22{ }^{\circ} \mathrm{C}$ in the single-photon timing system as previously described (17). ShB and ShB-L7E peptides were excited at $285 \mathrm{~nm}$ through a frequency doubled, cavity dumped (3.7 $\mathrm{MHz}$ repetition rate) dye laser of Rhodamine 6G (Coherent 701-2), synchronously pumped by a mode-locked $\mathrm{Ar}^{+}$laser (514.5 nm, Coherent Innova 400-10). The emission was detected by a Hamamatsu R-2809 MCP photomultiplier at 320 nm (Jobin-Yvon HR320 monochromator). The instrumental response function ( $80 \mathrm{ps}$ ) was generated from a scatter dispersion (silica, colloidal water solution). The time scaling was 11 ps per channel, and 1024 channels were in use.
The kinetic parameters of the decay of the fluorescence intensity (lifetimes, $\tau_{i}$, and normalized amplitudes, $\alpha_{i}$ ) were determined using nonlinear least-squares regression methods. The lifetime-weighted quantum yield, $\bar{\tau}$ (18), was determined according to

$$
\bar{\tau}=\sum \alpha_{i} \tau_{i}
$$

and the average fluorescence lifetime was calculated by (18)

$$
\langle\tau\rangle=\frac{\sum \alpha_{i} \tau_{i}^{2}}{\sum \alpha_{i} \tau_{i}}
$$

The anisotropy decay parameters (rotational correlation times, $\varphi_{i}$, amplitudes, $\beta_{i}$, and residual anisotropy, $r_{\infty}$ ) were determined using a nonlinear least-squares global analysis method by simultaneously fitting the vertically and horizontally polarized emission components to a sum of $n$ exponentials and a constant term $(19,20)$ :

$$
r(t)=\left(r(0)-r_{\infty}\right)\left[\sum_{i=1}^{n} \beta_{i} \exp \left(-t / \phi_{i}\right)\right]+r_{\infty}
$$

where $\sum_{i=1}^{n} \beta_{i}=1$. In both cases, the fits tabulated here represent the minimum set of adjustable parameters that satisfy the usual statistical criteria, namely, a reduced $\chi^{2}$ value of $<1.3$ and a random distribution of weighted residuals.

Quenching Experiments. Stock solutions of the quencher (5NS and $16 \mathrm{NS}$ ) were prepared in ethanol at $7.8 \times 10^{-4} \mathrm{M}$. Aliquots of these solutions were directly added to a cuvette containing the suspension of PA LUVs/peptide, and the quenching of tyrosine fluorescence was recorded and analyzed by Stern-Volmer plots $\left[I_{0} / I_{\mathrm{q}}\right.$ versus the quencher concentration in the lipid phase $\left.\left([\mathrm{Q}]_{\mathrm{L}}\right)\right]$. If $K_{\mathrm{PQ}}=[\mathrm{Q}]_{\mathrm{L}} /[\mathrm{Q}]_{\mathrm{W}}$ is the molar partition coefficient of the quencher between the phospholipid and the aqueous phases, $[\mathrm{Q}]_{\mathrm{L}}$ is given by

$$
[\mathrm{Q}]_{\mathrm{L}}=\frac{K_{\mathrm{PQ}} V_{\mathrm{T}}}{V_{\mathrm{W}}+V_{\mathrm{L}} K_{\mathrm{PQ}}}[\mathrm{Q}]_{\mathrm{T}}
$$

where $[\mathrm{Q}]_{\mathrm{T}}$ is the concentration of quencher in the total volume $V_{\mathrm{T}}\left(V_{\mathrm{T}}=V_{\mathrm{L}}+V_{\mathrm{W}}\right)$ and $V_{\mathrm{L}}$ and $V_{\mathrm{W}}$ are respectively the volume of the lipid and aqueous phases (see ref 21). For $5 \mathrm{NS}$ and $16 \mathrm{NS}, K_{\mathrm{PQ}}$ in the fluid phase is $8.9 \times 10^{4}$ and 9.73 $\times 10^{3}$, respectively (22), and for PA, a phospholipid molar volume $\left(V_{\mathrm{L}}\right)$ of $0.7 \mathrm{M}^{-1}$ was used (23).

Energy Transfer Experiments. For energy transfer experiments the fluorescent probe trans-parinaric acid ( $t$-PnA) was used as an acceptor of the peptide tyrosine excitation. The Förster radius for energy transfer, $R_{0}$, was determined from (18)

$$
R_{0}=0.2108\left(\kappa^{2} \Phi_{\mathrm{F}} n^{-4} J\right)^{1 / 6}
$$

where $J$, the overlap integral, was evaluated by the integration described in eq 6 between the normalized donor emission spectrum, $I(\lambda)$, and the acceptor absorption spectrum, $\epsilon(\lambda)$ (in $\mathrm{M}^{-1} \mathrm{~cm}^{-1}$ units), as defined by

$$
J=\int_{0}^{\infty} I(\lambda) \epsilon(\lambda) \lambda^{4} \mathrm{~d} \lambda
$$

In eqs 5 and $6, \kappa^{2}$ is the orientation factor, $\Phi_{\mathrm{F}}$ the quantum 
yield of the donor in the absence of acceptor, and $n$ the refractive index of the medium. $R_{0}$ is expressed in angstroms and $\lambda$, the wavelength, in nanometers. Fixed values for $\kappa^{2}$ $=2 / 3$ (corresponding to a dynamic isotropic regime of energy transfer) and $n=1.425$ (24) were used in eq 5.

Energy transfer efficiencies were determined from the equation:

$$
E=1-I^{\mathrm{DA}} / I^{\mathrm{D}}
$$

where $I^{\mathrm{DA}}$ and $I^{\mathrm{D}}$ are the fluorescence intensities in the presence and absence of acceptor, respectively. Increasing aliquots of the acceptor, $t$-PnA in ethanol, were added to a cuvette containing the suspension of PA LUVs/peptide at $22{ }^{\circ} \mathrm{C}$, and the decrease in tyrosine fluorescence was monitored upon excitation at $275 \mathrm{~nm}$.

In a steady-state study of energy transfer, eventual artifacts should be taken into account. In this way, the effect of the acceptor ( $t$-PnA) absorption at the donor (tyrosine) excitation wavelength $(275 \mathrm{~nm})$ was corrected according to the equation:

$$
I_{\text {corr }}^{\mathrm{D}}=I_{\exp }^{\mathrm{D}}\left(\frac{A_{\mathrm{T}}}{A_{\mathrm{D}}}\right)\left(\frac{1-10^{-A_{\mathrm{D}}}}{1-10^{-A_{\mathrm{T}}}}\right)
$$

where $I_{\exp }^{\mathrm{D}}$ is the experimentally obtained value and $A_{\mathrm{D}}$ and $A_{\mathrm{T}}$ are the absorbances for the donor and total solution (donor and acceptor), respectively. Also, the correction for the acceptor absorption of the donor emission (trivial process or reabsorption) was carried out as described by the equation:

$$
I_{\text {corr }}^{\mathrm{D}}=I_{\exp }^{\mathrm{D}} \frac{2.303 A_{\mathrm{A}}}{1-10^{-A_{\mathrm{A}}}}
$$

where $A_{\mathrm{A}}$ is now the absorption of the acceptor at the emission wavelength of the donor $(320 \mathrm{~nm})$.

\section{RESULTS}

Steady-State Fluorescence of ShB and ShB-L7E Peptides in Aqueous Solution. Fluorescence excitation and emission spectra of ShB and ShB-L7E in buffer are shown in Figure 1. The excitation maxima $(277 \mathrm{~nm})$ are slightly red shifted compared to those of free tyrosine $(274 \mathrm{~nm})$, suggesting that in both peptides tyrosine is located in a relatively hydrophobic environment (25). The emission spectra show the characteristic emission band for tyrosine with maxima at 303 $\mathrm{nm}$, which remain unaltered at $\mathrm{pH}$ ranging from 5 to 10 (data not shown). Since the emission spectra do not show any shoulder around $340 \mathrm{~nm}$ in the $\mathrm{pH}$ range studied, the possible ionization or strong hydrogen binding of the phenol hydroxyl group of tyrosine can be discarded. Fluorescence quantum yields measured using 5-methoxyindole as a reference (26) were 0.05 and 0.04 for $\mathrm{ShB}$ and ShB-L7E, respectively (Table 1). These values were considerably lower than that of free tyrosine $[\Phi=0.14(27)]$, in reasonable agreement with values obtained for other peptides and proteins (2830 ). The steady-state fluorescence anisotropy measured in buffer at $22{ }^{\circ} \mathrm{C}$ by exciting at $285 \mathrm{~nm}$ was relatively similar for both peptides $(\langle r\rangle=0.039$ and $\langle r\rangle=0.051$ for ShB and ShB-L7E, respectively) and much smaller than the intrinsic anisotropy $\left(r_{0}=0.29\right)$ corresponding to immobilized tyrosines at that excitation wavelength (31).
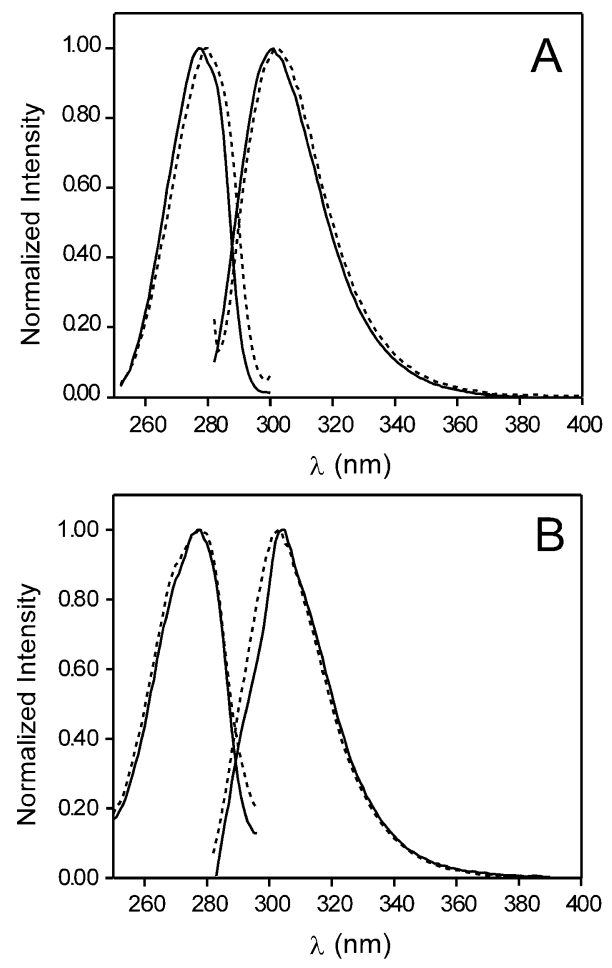

FIGURE 1: Normalized and corrected excitation and emission fluorescence spectra of ShB peptide (panel A) and mutant ShBL7E peptide (panel B) in $10 \mathrm{mM}$ Hepes, $\mathrm{pH}$ 6, and $100 \mathrm{mM} \mathrm{NaCl}$ buffer (-) and in PA LUVs in the same buffer (--). The temperature was maintained at $22{ }^{\circ} \mathrm{C}$.

Time-Resolved Fluorescence of ShB and ShB-L7E Peptides in Aqueous Solution. Fluorescence decay of the peptides was best fitted to triexponential functions (Table 1). Fluorescence fit parameters were rather similar for both peptides, and only small differences could be detected for the intermediate and long lifetime components. Since $\bar{\tau}$ (eq 1) is proportional to the quantum yield in the absence of static contributions, the values in Table 1 contain information about the eventual existence of such phenomenon. In fact, $\bar{\tau}$ is identical for both peptides $(\bar{\tau}=1.4 \mathrm{~ns})$, but the quantum yield as determined from steady-state measurements is slightly higher for ShB. This suggests the presence of a static fluorescence quenching process in the mutant peptide in which the glutamic acid at position 7 could be involved.

In the time-resolved anisotropy experiments, at least two rotational correlation times were needed to describe the decay processes, which were similar for both peptides (Table 2). Since the long rotational correlation time $\left(\varphi_{2}\right)$ was five times higher than the short one $\left(\varphi_{1}\right)$, the total anisotropy can be interpreted as the product of two independent depolarizing processes, a first one due to fast movements of the peptide segment containing the tyrosine residue $\left[r^{\prime}(t)\right]$ and a second one related to the global rotational motion of the whole peptide (32):

$$
\begin{gathered}
r(t)=r^{\prime}(t) \mathrm{e}^{-t / \varphi_{\text {global }}} \\
r^{\prime}(t)=r(0)\left[\left(1-S_{1}{ }^{2}\right) \mathrm{e}^{-t / \varphi_{\text {segmental }}}+S_{1}{ }^{2}\right]
\end{gathered}
$$

where $S_{1}$ is the order parameter characterizing the internal fluctuation of the peptide segment containing the tyrosine residue, and the short and long rotational correlation times 
Table 1: Photophysical Parameters of ShB and ShB-L7E (Quantum Yield, $\Phi$, Fluorescence Lifetimes, $\tau_{i}$, and Normalized Amplitudes $\alpha_{i}$,) in Aqueous Buffer and Incorporated into PA Vesicles at $22{ }^{\circ} \mathrm{C}$

\begin{tabular}{llcccccccccc}
\hline peptide & medium & $\Phi$ & $\alpha_{1}$ & $\tau_{1}(\mathrm{~ns})$ & $\alpha_{2}$ & $\tau_{2}(\mathrm{~ns})$ & $\alpha_{3}$ & $\tau_{3}(\mathrm{~ns})$ & $\chi^{2}$ & $\bar{\tau}^{a}$ & $\langle\tau\rangle^{b}$ \\
\hline ShB & buffer & 0.05 & 0.26 & 0.4 & 0.55 & 1.4 & 0.19 & 3.0 & 1.1 & 1.4 & 2.0 \\
ShB-L7E & buffer & 0.04 & 0.27 & 0.4 & 0.55 & 1.2 & 0.18 & 3.6 & 1.0 & 1.4 & 2.2 \\
ShB & PA & 0.05 & 0.32 & 0.4 & 0.51 & 1.5 & 0.17 & 3.7 & 1.1 & 1.5 & 2.3 \\
ShB-L7E & PA & 0.06 & 0.32 & 0.4 & 0.47 & 1.4 & 0.21 & 5.7 & 1.1 & 2.0 & 3.9 \\
\hline
\end{tabular}

${ }^{a}$ Determined from eq $1 .{ }^{b}$ Determined from eq 2.

Table 2: Time-Resolved Fluorescence Anisotropy Parameters of ShB and ShB-L7E (Rotational Correlation Times, $\varphi_{i}$, Amplitudes, $\beta_{i}$, and Residual Anisotropy, $r_{\infty}$ ) in Aqueous Buffer and Incorporated into PA Vesicles at $22{ }^{\circ} \mathrm{C}$

\begin{tabular}{llccccccc}
\hline \multicolumn{1}{c}{ peptide } & medium & $r(0)$ & $\beta_{1}$ & $\begin{array}{c}\varphi_{1} \\
(\mathrm{~ns})\end{array}$ & $\beta_{2}$ & $\begin{array}{c}\varphi_{2} \\
(\mathrm{~ns})\end{array}$ & $r_{\infty}$ & $\chi^{2}$ \\
\hline ShB & buffer & 0.20 & 0.51 & 0.2 & 0.49 & 0.9 & 0 & 1.0 \\
ShB-L7E & buffer & 0.20 & 0.67 & 0.2 & 0.33 & 0.9 & 0 & 1.0 \\
ShB & PA & 0.17 & 0.42 & 0.2 & 0.58 & 4.3 & 0.07 & 1.0 \\
ShB-L7E & PA & 0.18 & 0.64 & 0.1 & 0.36 & 0.3 & 0.06 & 1.1 \\
\hline
\end{tabular}

Table 3: Parameters from the Fit of an Independent Two-Motion Model (Eqs 10, 11, and 15) to the Anisotropy Decay of ShB and ShB-L7E (Segmental and Global Correlation Times, $\varphi_{i}$, Order Parameters, $S_{i}$, and Cone Angles, $\left.\theta_{0 i}\right)$ in Aqueous Buffer and Incorporated into PA Vesicles at $22{ }^{\circ} \mathrm{C}$

\begin{tabular}{lccccccc}
\hline peptide & medium & $\begin{array}{c}\varphi_{\text {seg }} \\
(\mathrm{ns})\end{array}$ & $\begin{array}{c}\varphi_{\text {global }} \\
(\mathrm{ns})\end{array}$ & $S_{1}$ & $S_{2}$ & $\begin{array}{c}\theta_{01} \\
(\mathrm{deg})\end{array}$ & $\begin{array}{c}\theta_{02} \\
(\mathrm{deg})\end{array}$ \\
\hline ShB & buffer & 0.2 & 0.9 & 0.70 & 0 & 38 & \\
ShB-L7E & buffer & 0.2 & 0.9 & 0.57 & 0 & 47 & \\
ShB & PA & 0.2 & 4.3 & 0.87 & 0.75 & 24 & 35 \\
\hline
\end{tabular}

obtained from the fit are related to $\varphi_{\text {global }}$ and $\varphi_{\text {segmental }}$ (Table 3) by

$$
\begin{gathered}
\varphi_{2}=\varphi_{\text {global }} \\
\varphi_{1}=\frac{\varphi_{\text {segmental }} \varphi_{\text {global }}}{\varphi_{\text {segmental }}+\varphi_{\text {global }}}
\end{gathered}
$$

Binding of ShB and ShB-L7E Peptides to Lipid Membranes. The interaction of ShB and ShB-L7E peptides with lipid membranes was studied using PA vesicles. The quantum yield of ShB remained unchanged when LUVs of PA were added to the aqueous solution whereas for ShB-L7E this parameter increased 1.5 times upon addition of the vesicles (Table 1). Fluorescence spectra were similar to those obtained in buffer (Figure 1), and only a slight red shift was observed in the excitation and emission spectra of ShB upon incorporation in the membrane, suggesting a more hydrophobic environment surrounding the tyrosine residue. On the other hand, a large increase in the steady-state fluorescence anisotropy of both peptides was observed upon vesicle addition, so this parameter was selected to determine the phospholipid/water molar partition coefficient, $K_{\mathrm{P}}$ :

$$
K_{\mathrm{P}}=\frac{n_{\mathrm{L}} / V_{\mathrm{L}}}{n_{\mathrm{W}} / V_{\mathrm{W}}}
$$

where $n_{\mathrm{i}}$ stands for moles of peptide in phase $\mathrm{i}$ and $V_{\mathrm{i}}$ for volume of phase $i$. The phase is either aqueous $(i=W)$ or lipidic $(\mathrm{i}=\mathrm{L})$. The relationship and assumptions used to

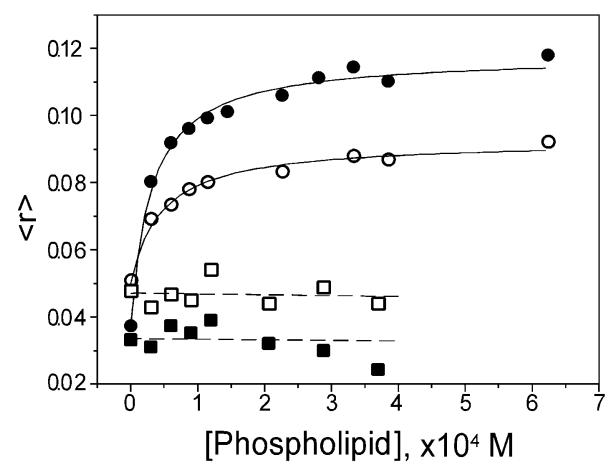

FIGURE 2: Steady-state anisotropy of tyrosine from ShB peptide $(\boldsymbol{\square}, \mathbf{\square})$ and from mutant ShB-L7E peptide $(O, \square)$ as a function of PA $(\bullet, \bigcirc)$ and PC $(\square, \square)$ concentration. Excitation and emission wavelengths were 285 and $320 \mathrm{~nm}$, respectively. The solid lines are the fits of eq 13 to the data. The dotted lines are just a guide to the eye. The temperature was maintained at $22{ }^{\circ} \mathrm{C}$.

determine $K_{\mathrm{P}}$ from the steady-state anisotropy are given by (33)

$$
\langle r\rangle=\frac{r_{\mathrm{W}} D+r_{\mathrm{L}} Y K_{\mathrm{P}}}{Y K_{\mathrm{P}}+D}
$$

in which $r_{\mathrm{W}}$ and $r_{\mathrm{L}}$ are the steady-state anisotropies in the aqueous and lipid phases, respectively; $Y=\Phi_{\mathrm{L}} / \Phi_{\mathrm{W}}$ is the ratio of the fluorescence quantum yield of the peptide in the lipid and aqueous phase; and $D=(1 / \gamma[\mathrm{L}])-1$ is a function of $[\mathrm{L}]$, the lipid concentration, and $\gamma$, the molar volume of the lipid $\left(0.7 \mathrm{M}^{-1}\right)$.

Figure 2 shows the increase in $\langle r\rangle$ upon addition of PA LUVs. From a two-parameter $\left(r_{\mathrm{L}}\right.$ and $\left.K_{\mathrm{P}}\right)$ fitting procedure (eq 13), partition coefficients of $K_{\mathrm{P}}=(4.5 \pm 0.5) \times 10^{4}$ and $K_{\mathrm{P}}=(2.2 \pm 0.3) \times 10^{4}$ and anisotropy values of $r_{\mathrm{L}}=$ $0.120 \pm 0.003$ and $r_{\mathrm{L}}=0.093 \pm 0.003$ were obtained for $\mathrm{ShB}$ and ShB-L7E, respectively.

The binding of ShB and ShB-L7E to PC vesicles was also studied through anisotropy measurements. For both peptides $\langle r\rangle$ remained invariant upon lipid addition (Figure 2).

Studies of the Transverse Location of the Peptides in the Bilayer. To obtain information on the location of both peptides in the lipid bilayer, both fluorescence quenching and energy transfer approaches were used. The differential quenching of the fluorescence of both peptides by the spin probes 5NS and 16NS was measured in PA LUVs. The corresponding Stern-Volmer plots are shown in Figure 3. Since in these spin probes the nitroxide group is located at 12 and $3 \AA$, respectively, from the bilayer center (34), the direct comparison of the plots provides a qualitative method to determine the transverse location of both peptides within the bilayer. Quenching by 5NS was small and similar for both peptides, and no definitive conclusion can be made on their membrane topology. At variance, use of 16NS shows 

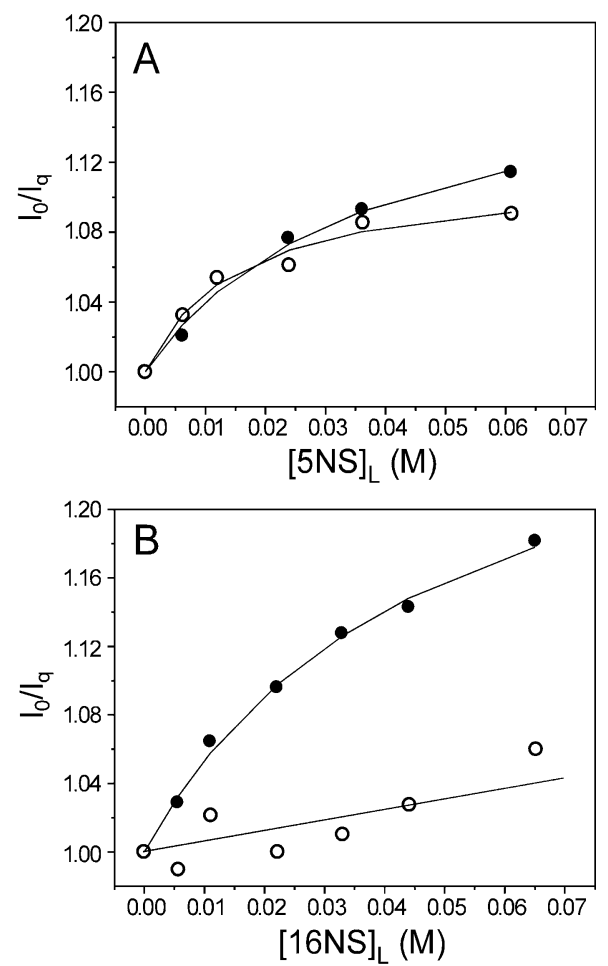

FIGURE 3: Stern-Volmer plots for the quenching of tyrosine from ShB peptide $(0)$ and from mutant ShB-L7E peptide $(O)$ by $5 \mathrm{NS}$ (panel A) and 16NS (panel B) incorporated into LUVs of PA at 22 ${ }^{\circ} \mathrm{C}$. The lines are just a guide to the eye. Excitation and emission wavelengths were 275 and $320 \mathrm{~nm}$, respectively.

a higher quenching efficiency for $\mathrm{ShB}$ as compared to ShBL7E. Therefore, a shallower membrane position for the latter peptide can be concluded from these experiments. The quenching efficiencies were much lower than those observed for other phenol derivatives $(35,36)$, and all of the plots, with the exception of ShB-L7E quenched by 16NS, were nonlinear, showing a downward curvature. This could be due to the existence of a significant peptide fraction, nonaccessible to the lipophilic quenchers, in the aqueous phase. However, as estimated from the partition coefficients, under our experimental conditions only 2\% (ShB) and 5\% (ShBL7E) of the peptides were present in the aqueous phase. Taking this into account, we interpret the observed downward curvature on the basis of a more complex accessibility of the quenchers to the membrane-bound peptide as compared to the peptide in solution. This should prevent a data analysis in the context of the parallax model formalism (34) in order to determine quantitatively the tyrosine transverse location.

To further support the above topological conclusion, the relative location of the peptides within the membrane was also addressed from energy transfer experiments, using the fluorescent probe $t$-PnA as acceptor of the peptide tyrosine excitation. The calculated Förster radius values for $\mathrm{ShB}$ and ShB-L7E were $R_{0}=25.8 \pm 0.2 \AA$ and $R_{0}=26.4 \pm 0.2 \AA$, respectively. Figure 4 illustrates the energy transfer efficiency between the peptides and the $t$-PnA (eq 7) as a function of $C$, the number of acceptors per $R_{0}^{2}$ [calculated assuming an area of $70 \AA^{2}$ per lipid molecule (23)]. As shown, changes in the energy transfer efficiency for $\mathrm{ShB}$ are in full agreement with the theoretical expectation for membrane bidimensional random distribution of donors and acceptors within the same plane (37). The acceptor ( $t$-PnA) is known to be internalized

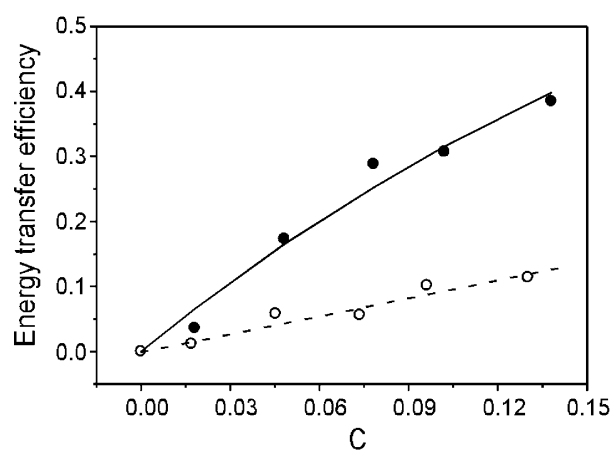

FIGURE 4: Energy transfer efficiency between $\mathrm{ShB}$ peptide $(0)$ or mutant ShB-L7E peptide $(O)$ and $t$-PnA, as a function of $C$, the number of energy transfer acceptors $(t-\mathrm{PnA})$ per $R_{0}{ }^{2}$. The solid line is the theoretical expectation for bidimensional random distribution of acceptors in the same plane (see text). The dotted line is just a guide to the eye. The temperature was $22^{\circ} \mathrm{C}$.

in the membrane (38), and from molecular models a distance of about $12.1 \AA$ to the aqueous interface has been estimated (39). In this way, it can be concluded that tyrosine in $\mathrm{ShB}$ is located into the membrane at this depth. At variance, a much lower energy transfer is obtained for ShB-L7E, which can be rationalized on the basis of a significant interplanar distance between the ShB-L7E donor and acceptor. Upon integration of the decay law for this geometry (40), it is concluded that an interplanar distance of about $12 \AA$ describes the experimental data (fit not shown). This result indicates that, at variance with ShB, tyrosine in ShB-L7E is located near the lipid-water interface.

Time-Resolved Fluorescence of ShB and ShB-L7E Peptides in Lipid Membranes. The decay of the fluorescence emission of the peptides in PA vesicles was clearly triexponential (Table 1). For ShB the short and intermediate lifetimes were similar to those obtained in buffer, and only a slight increase was observed in the longest component. However, a more significant change was observed in the fluorescence kinetics of ShB-L7E, where $\tau_{2}$ and especially $\tau_{3}$ increased upon membrane incorporation. As previously described, these data are not biased due to a significant fraction of peptides in buffer.

Representative experimental decay curves are shown in Figure 5, where it can be seen that the anisotropy decays for both peptides with different kinetics to reach a residual, time-independent value different from zero. The results of the deconvolution analysis of the experimental data are listed in Table 2. For ShB, at least two rotational correlation times were needed to describe the decay process: a subnanosecond component $(0.2 \mathrm{~ns})$ similar to the short one obtained in buffer and a second component $(4.3 \mathrm{~ns})$, clearly slower than that in buffer $(0.9$ ns). For ShB-L7E, two rotational correlation times were also needed to describe the anisotropy kinetics, both very short and in the same time scale ( 0.1 and $0.3 \mathrm{~ns}$ ). The residual anisotropy value $r_{\infty}$, indicative of a restricted motion of the tyrosine side chain on the time scale of the fluorescence experiment, was slightly higher for ShB than for ShB-L7E. Since the two correlation times of ShB differ by more than an order of magnitude, they are assumed to reflect two independent molecular motions. Following the model previously used for the anisotropy experiments in solution, the total anisotropy was interpreted as the product of two independent depolarizing processes, one due to fast 

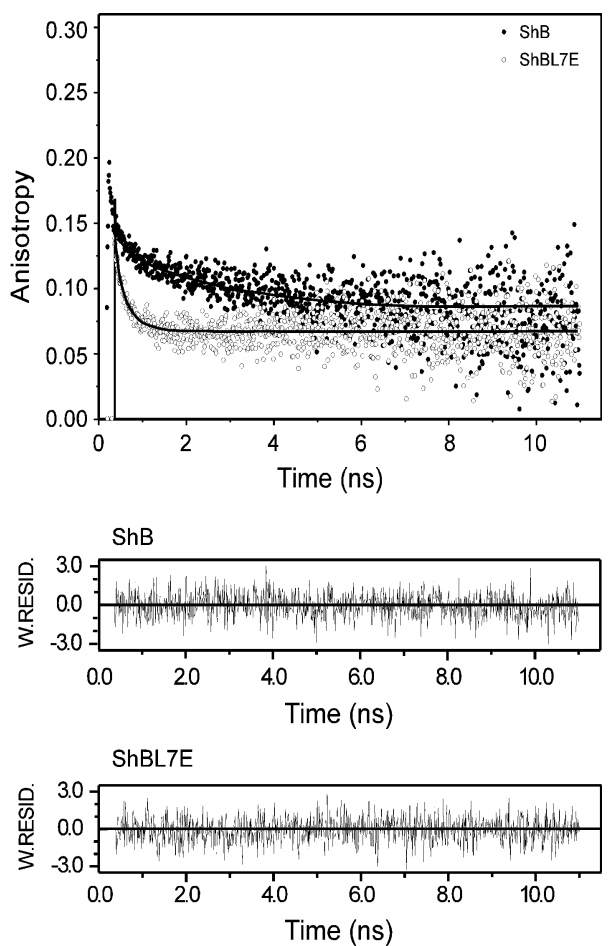

FIGURE 5: Time-resolved fluorescence anisotropy of tyrosine from ShB peptide and mutant ShB-L7E peptide in LUVs of PA at 22 ${ }^{\circ} \mathrm{C}$. Experimental data and that from the corresponding fit (see Table 2 for decay parameters) are shown as points and as a continuous line, respectively. Residuals of each fit are also shown. Excitation and emission wavelengths were 285 and $320 \mathrm{~nm}$, respectively.

movements of the peptide segment containing the tyrosine residue $\left[r^{\prime}(t)\right]$ and the other related to the global rotational motion of the whole peptide into the lipid bilayer (32):

$$
\begin{gathered}
r(t)=r^{\prime}(t)\left[\left(1-S_{2}^{2}\right) \mathrm{e}^{-t / \varphi_{\text {global }}}+S_{2}^{2}\right] \\
r^{\prime}(t)=r(0)\left[\left(1-S_{1}^{2}\right) \mathrm{e}^{-t / \varphi_{\text {segmental }}}+S_{1}^{2}\right]
\end{gathered}
$$

where $S_{1}$ and $S_{2}$ are the order parameters characterizing the internal and the whole peptide fluctuations. Since $\varphi_{2} \gg \varphi_{1}$, the above expression can be simplified to the sum of two exponential functions plus a constant term:

$$
\begin{aligned}
& r(t)=r(0)\left[\left(1-S_{1}{ }^{2}\right) \exp \left(-t / \varphi_{\text {segmental }}\right)+\right. \\
& \left.\left(1-S_{2}{ }^{2}\right) S_{1}{ }^{2} \exp \left(-t / \varphi_{\text {global }}\right)+S_{1}{ }^{2} S_{2}{ }^{2}\right]
\end{aligned}
$$

and the experimental rotational correlation times, $\varphi_{1}$ and $\varphi_{2}$, can be directly related to $\varphi_{\text {segmental }}$ and $\varphi_{\text {global }}$, respectively. These parameters are shown in Table 3, together with the order parameters $S_{1}$ and $S_{2}$.

\section{DISCUSSION}

The recent publication of the high-resolution structure of the potassium prokaryote channels KcsA (41) and MthK (42) and, especially, its recombinant production and functional reconstitution into lipid vesicles open the possibility of using such channels to study the interactions between rapidly inactivating peptides and their hydrophobic binding sites on the cytoplasmic side of open channels. Nonetheless, the use of anionic lipid vesicles as the inactivating peptide target, mimicking properties of the channel pore, has proven to be an useful and simple model that has allowed to establish a correlation between the adoption of a hydrophobic $\beta$-hairpin structure by the ShB peptide and its ability to inactivate the channel $(10,12-14)$. In this way, modifications at the ShB $\mathrm{N}$-terminal moiety that impede the adoption of the $\beta$-hairpin in lipid membranes, as is the case of the L7E mutation or the phosphorylation of the Y8 residue, result in noninactivating peptide variants.

The ShB and ShBL7E peptides contain a single tyrosine residue (and no tryptophan), so we have exploited its photophysical properties to obtain additional information about the affinity, location, orientation, and conformational dynamics of these peptides in anionic membranes. For this purpose we first analyzed the photophysical and dynamic properties of the peptides in solution as a prerequisite to understand their interactions with the phospholipid systems. The small red shifts observed on the excitation fluorescence spectra of both ShB and ShB-L7E in buffer are compatible with the existence of a hydrophobic environment around the tyrosine residue, which could be supplied by the surrounding nonpolar amino acids present at the $\mathrm{NH}_{2}$-terminal half of the peptide. Only small differences were found in the quantum yield and fluorescence lifetimes of both peptides, which could be ascribed to the presence in ShB-L7E of the glutamic acid close to the tyrosine residue. Direct intramolecular interaction between the carbonyl group of the glutamic acid and the tyrosine aromatic ring could be the cause of these differences, pointing out to a static fluorescence quenching component. However, this would not imply a proton transfer, since it has been shown (10) that the glutamic acid of ShB-L7E remains essentially protonated at neutral $\mathrm{pH}$ and, therefore, cannot act as a proton acceptor for the excited tyrosine. This hypothesis is also ruled out from our experiments since tyrosinate emission was not observed in the ShB-L7E fluorescence spectrum even at $\mathrm{pH}$ 10.

The triexponential behavior of the fluorescence decay observed in both peptides has been similarly reported for other peptides and proteins with a single tyrosine residue $(16,30,31,43,44)$ and reflects the existence of groundstate rotamers sensing different chemical environments, which interconvert slowly on the nanosecond time scale (45, 46). The small differences observed in the intermediate and longest lifetimes of ShB-L7E compared to those of ShB should correspond to differences in the local interactions sensed by two of the three rotamers due to the presence of the glutamic acid.

Similar dynamic properties were observed for ShB and ShB-L7E from time-resolved depolarization experiments. The longest rotational correlation time $(0.9 \mathrm{~ns}$ for both peptides) should reflect the global motion of the peptide. Assuming spherical symmetry for the peptides, the equivalent hydrodynamic radius, $a=(3 k T \phi /(4 \pi \eta))^{1 / 3}$ (where $\eta$ is the solution viscosity), should be $9.6 \AA$. A radius of $9.4 \AA$ was obtained from the partial specific volume of the peptides $(0.54 \mathrm{~mL} / \mathrm{g})$, assuming a fraction of hydration of $0.4 \mathrm{~g}$ of water/g of peptide (47). The good agreement between the two values indicates that both peptides essentially remain in the monomeric state in solution at the concentrations studied.

The segmental correlation time $(0.2 \mathrm{~ns})$ reflects fast movements of, at least, one peptide segment containing the tyrosine residue, which is more restricted in $\mathrm{ShB}$ than in $\mathrm{ShB}$ L7E, because of the higher $S_{1}$ value obtained for ShB. The 
angular displacement of these motions can be extracted from $S_{1}$ assuming a "wobbling in a cone" model (48). Following this model, the cone angle $\theta_{01}$ in which the segment containing tyrosine rotates is given by $\cos \theta_{01}=1 / 2\left[\left(8 S_{1}+\right.\right.$ $\left.1)^{1 / 2}-1\right]$. Angles of $38^{\circ}$ and $47^{\circ}$ were obtained for $\mathrm{ShB}$ and ShB-L7E, respectively (Table 3), and suggest that this segment experiences relatively large angular displacement during the tyrosine lifetime. The $r(0)$ value obtained was lower than the 0.29 value expected for an immobilized tyrosine residue upon excitation to $285 \mathrm{~nm}$ (31). An ultrafast energy homotransfer between tyrosines of different peptides could not be responsible for this initial depolarization since the peptides remain in solution as monomers, as shown before. The existence of an additional ultrafast motion on the subpicosecond/picosecond time scale that cannot be observed with our instrument is likely to be one source of this depolarization, altogether with possible fast electronic relaxation processes.

As concluded from the partition coefficients both $\mathrm{ShB}$ and ShB-L7E interact efficiently with anionic phospholipids and do not show a significant affinity for zwitterionic lipids, as was reported previously from infrared and DSC experiments and from fluorescence experiments using NBD-labeled peptides (12). Only small differences among the partition coefficients obtained in the present work and those reported previously (12) were found, which should be ascribed to effects of NBD labeling, which could partially modify the affinity of the peptides for the membrane.

Although an electrostatic interaction is expected to be responsible for the high affinity of ShB and ShB-L7E for the membrane, the final location of both peptides into the membrane should be different, since different steady-state anisotropy values were obtained in each case. In fact, from the partition coefficient study (Figure 2), values of $\langle r\rangle=$ 0.120 for ShB and $\langle r\rangle=0.093$ for ShB-L7E were obtained from the fit of eq 13 to the experimental data. The higher value obtained for $\mathrm{ShB}$ could suggest that tyrosine locates in a more ordered environment, probably distant from the phospholipid-water interface. The observed increase in the ShB-L7E quantum yield and fluorescence lifetimes upon interaction with membranes could indicate that its tyrosine is located within the bilayer in a deeper region than that of $\mathrm{ShB}$, but this behavior could also be expected if tyrosine remains close to the bilayer surface, where new interactions can be established between amino acids and phospholipid polar heads. These interactions could decrease the ability of the glutamic acid to quench tyrosine fluorescence. The rest of the experimental results support this latter interpretation; i.e., $\mathrm{ShB}$ is buried deep within the membrane, while ShBL7E remains in a shallower location, close to the phospholipid-water interface. This different location is evidenced by (i) the results of the differential quenching study which show that 16NS quenches ShB fluorescence more effectively than 5NS, but slightly quenches ShB-L7E fluorescence, (ii) the energy transfer study which indicates that ShB locates its tyrosine at $\sim 12 \AA$ from the phospholipid surface, while the ShB-L7E tyrosine remains close to the lipid-water interface, and (iii) the steady-state and time-resolved fluorescence anisotropy experiments, which indicate that $\mathrm{ShB}$ tyrosine is located in a much more viscous and ordered medium, compatible with the aliphatic region of a lipid bilayer.
The above results are in agreement with those reported in a previous work from freeze-fracture and infrared experiments (13), which show that ShB peptide inserts deeply enough into the vesicle bilayer as to perturb both hemilayers, thus suggesting that the adopted $\beta$-hairpin structure gets buried into the hydrophobic region, parallel to the phospholipid acyl chains. An additional C-terminal portion of the ShB peptide should remain lying over the membrane surface facilitating electrostatic interactions. According to this model, at least two rotational correlation times and a residual anisotropy must be extracted from the time-resolved fluorescence depolarization experiments of the $\mathrm{ShB}$ peptide inserted in membranes: a short correlation time which reflects internal fluctuations of the tyrosine residue, a longest component corresponding to fluctuations of the whole peptide segment incorporated into the membrane, and a residual value $r_{\infty}$ which shows that the reorientational motion of the tyrosine side chain is restricted on the time scale of the fluorescence experiment due to interactions with phospholipid chains. Assuming that the hairpin structure behaves like a rigid body into the bilayer, we could estimate the diffusion coefficient corresponding to the rigid body fluctuations from the longest rotational correlation time as $D_{\perp}=\sigma / \varphi_{\text {global }}(49,50)$, where $\sigma$ is a function of the order parameter $S_{2}$ and can be computed from the polynomial approximation described in ref 51. A value of $D_{\perp}=2 \times 10^{7} \mathrm{~s}^{-1}$ was calculated from this equation, which can be used to estimate the dimension and amino acids involved in the $\beta$-hairpin structure. The dependence of $D_{\perp}$ on molecular geometry, viscosity $(\eta)$, and temperature $(T)$ may be expressed by a modified Debye-Stokes-Einstein equation (52): $D_{\perp}=k_{\mathrm{B}} T / 6 \eta V g_{\perp}$, where $V$ is the hydrodynamic volume and $g_{\perp}$ is a dimensionless friction coefficient depending only on the molecular shape. In the case of cylindrical rotors, $g_{\perp}=2 p^{2} / 9\left(\ln p+\delta_{\perp}\right)$, where $\delta_{\perp}=-0.662$ $+0.917 p^{-1}-0.050 p^{-2}$ is a function of the length-todiameter ratio $p=L / 2 R(53)$. Modeling the hairpin as a cylinder with a diameter of $8 \AA$ and assuming a lipid viscosity of $\eta \approx 0.2 \mathrm{P}(54,55)$, a volume $V \approx 850 \AA^{3}$ was estimated, which would be in agreement with the volume of the $\mathrm{N}$-terminal $1-10$ amino acids in $\mathrm{ShB}$, corresponding to the hydrophobic half of the peptide. The $\beta$-hairpin structure would then fold near valine and glycine residues, at positions 5 and 6, as depicted in Figure 6. In this picture, the required $\beta$-turn has been formed by the $4-7$ (VAGL) tetrapeptide sequence establishing four intramolecular hydrogen bonds which stabilize the structure (13). The C-terminal portion of the peptide (rich in positive charged residues) is modeled parallel to the membrane surface and as extended as possible to facilitate its electrostatic interaction with the negatively charged phospholipids headgroups. Because of its larger degree of rotational freedom, the glycine residue at position 11 could act as a hinge, allowing the fluctuations of the hairpin structure into the lipid membrane. The amplitude of these fluctuations can be extracted from the order parameter $S_{2}=0.75$, assuming the wobbling in a cone model (48) (Table 3). An angular displacement of $35^{\circ}$ was obtained, which was smaller than the value reported in fluid membranes from electron paramagnetic resonance and fluorescence depolarization techniques for anchored rigid probes (for review, see ref 51) and helical polypeptides containing tryptophan (50). The small angle suggests that the restriction in the motion of the $\beta$-hairpin structure is due not only to 


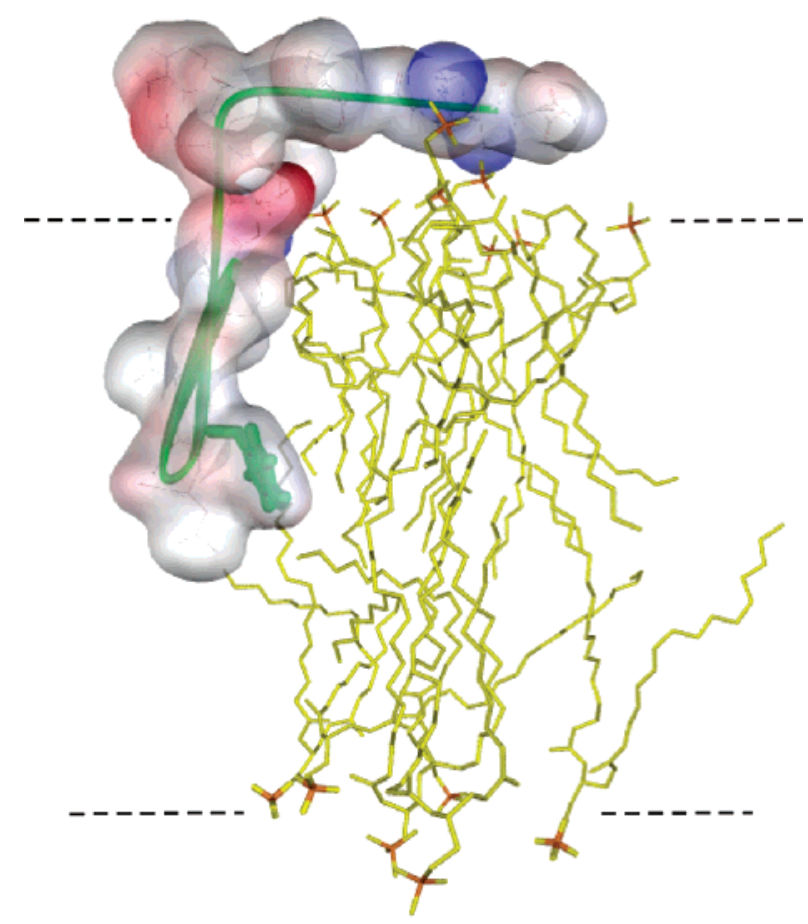

FIGURE 6: Model of the $\beta$-hairpin proposed for the ShB peptide inserted into palmitoyloleoylphosphatidic acid (POPA) vesicles. Modeling of the peptide and lipids was carried out on a Silicon Graphics workstation using the Sybyl program (Tripos Inc., St. Louis, MO). The resulting structures were submitted to an energy minimization procedure with the Sybyl force field. Phospholipids in the bilayer are colored in yellow. The peptide surface is colored according to the electrostatic potential (blue, electropositive, and red, electronegative charges). The $\beta$-structure backbone and Tyr- 8 residue are colored in green. The dotted lines represent the bilayer surface.

the phospholipid chains but also to intramolecular interactions with adjacent residues and to the strong electrostatic interactions established between the C-terminal portion of the peptide and the bilayer surface.

The short correlation time $(0.2 \mathrm{~ns})$ is similar to that obtained in buffer, suggesting that tyrosine is located in a fluid region, near the center of the bilayer, as concluded from the quenching and energy transfer experiments, and is shown in Figure 6. The angular displacement of this motion $\left(24^{\circ}\right)$, extracted from the order parameter $S_{1}=0.87$, was smaller than the value obtained in buffer $\left(38^{\circ}\right)$ and indicates that the hydrogen-bonded $\beta$-structure adopted by $\mathrm{ShB}$ within the membrane, as well as the proximity of the phospholipid acyl chains, restricts greatly the amplitude of the motion in the peptide segment containing tyrosine.

The dynamics of ShB-L7E in the membrane is different from that of ShB, exhibiting two very short rotational correlation times which could correspond to rotational motions of short segments containing tyrosine in a fluid medium. The residual anisotropy value also indicates that the amplitude of these motions is restricted due to intra- or intermolecular interactions. These results are compatible with those obtained from the quenching and energy transfer experiments and suggest that ShB-L7E interacts with the anionic phospholipid membranes but, due to the presence of the glutamic acid, it cannot traverse the surface and remains just grafted to it. The global rotational motion of the whole peptide is then prevented by interactions with the lipid surface, and only rapid fluctuations of the tyrosine side chain take place.

In conclusion, our results show that a wide variety of molecular details regarding the ShB peptide interaction with membranes can be obtained using the intrinsic fluorescence of its single tyrosine residue. From the photophysical properties of this fluorophore we have confirmed that ShB peptide is inserted into the anionic lipid membrane in a monomeric form to configure an intramolecular $\beta$-hairpin structure, while ShB-L7E remains grafted to the membrane surface. The location, size, and rotational dynamics of the $\beta$-hairpin from ShB have been estimated, and a model for the peptide inserted into the bilayer is proposed. This model is compatible with the recently reported crystal structure of the MthK channel (42), considered as a model for the open state of $\mathrm{K}^{+}$channels. The cytoplasmic vestibule of this channel, where the ShB peptide should stay during inactivation, is wide and long enough as to accommodate the $\beta$-hairpin proposed for the ShB. An additional model with a completely extended conformation has been proposed for the inactivating peptides interacting with the channel pore (56), although this study was done using another peptide (the $\beta 1$ subunit inactivation gate). According to our model, the hydrophobic half of ShB would be buried inside the channel cytoplasmic vestibule, occluding the pore and avoiding its interaction with water. The C-terminal half of the peptide, rich in positively charged residues, would stay out of the vestibule, possibly interacting with negatively charged amino acids at the rim of the cytoplasmic channel mouth.

\section{ACKNOWLEDGMENT}

We thank Drs. A. Fedorov and L. M. S. Loura (Centro de Química-Física Molecular, Lisboa) for their kind help with the time-resolved fluorescence measurements and data analysis, Drs. F. Gavilanes and J. P. Albar (Departamento de Bioquímica, Universidad Complutense, Madrid) for the synthesis and purification of ShB and ShB-L7E peptides, and Dr. G. Fernández-Ballester for help and comments on the computer modeling of the peptide.

\section{REFERENCES}

1. Hoshi, T., Zagotta, W. N., and Aldrich, R. W. (1990) Science 250, 533-538.

2. Zagotta, W. N., Hoshi, T., and Aldrich, R. W. (1990) Science 250, 568-571.

3. Isacoff, E. Y., Jan, Y. N., and Jan, L. Y. (1991) Nature 353, 8690.

4. Dubinsky, W. P., Mayorka-Wark, O., and Schultz, S. (1992) Proc. Natl. Acad. Sci. U.S.A. 89, 1770-1774.

5. Toro, L., Stefani, E., and LaTorre, R. (1992) Neuron 9, $237-$ 245.

6. Murrell-Lagnado, R. D., and Aldrich, R. W. (1993) J. Gen. Physiol. 102, 977-1003.

7. Armstrong, C. M., and Bezanilla, F. (1977) J. Gen. Physiol. 70, $567-590$

8. Durell, S. R., and Guy, H. R. (1992) Biophys. J. 62, 238-247.

9. Jan, L. Y., and Jan, Y. N. (1992) Annu. Rev. Physiol. 54, 537555 .

10. Fernández-Ballester, G., Gavilanes, F., Albar, J. P., Criado, C., Ferragut, J. A., and González-Ros, J. M. (1995) Biophys. J. 68, $858-865$.

11. Abbott, G. W., Mercer, E. A., Miller, R. T., Ramesh, B., and Srai, S. K. (1998) Biochemistry 37, 1640-1645.

12. Encinar, J. A., Fernández, A. M., Gavilanes, F., Albar, J. P., Ferragut, J. A., and González-Ros, J. M. (1996) Biophys. J. 71, $1313-1323$. 
13. Encinar, J. A., Fernández, A. M., Gil-Martín, E., Gavilanes, F., Albar, J. P., Ferragut, J. A., and González-Ros, J. M. (1998) Biochem. J. 331, 497-504.

14. Encinar, J. A., Fernández, A. M., Molina, M. L., Molina, A. Poveda, J. A., Albar, J. P., Lopez-Barneo, J., Gavilanes, F., FerrerMontiel, A. V., and Gonzalez-Ros, J. M. (2002) Biochemistry 41, $12263-12269$.

15. Gazit, E., and Shai, Y. (1993) Biochemistry 32, 1236312371.

16. Ferreira, S. T., Stella, L., and Gratton, E. (1994) Biophys. J. 66 , $1185-1196$

17. Loura, L. M., Fedorov, A., and Prieto, M. (1996) Biophys. J. 7, $1823-1836$

18. Lakowicz, J. R. (1999) in Principles of Fluorescence Spectroscopy, 2nd ed., Kluwer Academic/Plenum Press, New York.

19. Dale, R. E., Chen, L. A., and Brand, L. (1977) J. Biol. Chem. 252, 2163-2169.

20. Heyn, M. P. (1989) Methods Enzymol. 172, 462-471.

21. Castanho, M., and Prieto, M. (1995) Biophys. J. 69, 155-168.

22. Wardlaw, J. R., Sawyer, W. H., and Ghiggino, K. P. (1987) FEBS Lett. 231, 20-24.

23. Marsh, D. (1990) in Handbook of Lipid Bilayers, CRC Press, Boca Raton, Fl.

24. Toptygin, D., and Brand, L. (1995) J. Fluoresc. 5, 39-50.

25. Li, Y. K., Kuliopulos, A., Mildvan, A. S., and Talalay, P. (1993) Biochemistry 32, 1816-1824.

26. Demas, J. N., and Crosby, G. A. (1971) J. Phys. Chem. 75, 9911024.

27. Chen, R. F. (1967) Anal. Lett. 1, 35-42.

28. Ross, J. B., Laws, W. R., Buku, A., Sutherland, J. C., and Wyssbrod, H. R. (1986) Biochemistry 25, 607-612.

29. Härd, T., Hsu, V., Sayre, M. H., Geiduschek, E. P., Appelt, K., and Kearns, D. R. (1989) Biochemistry 28, 396-406.

30. Wu, P., Li, Y. K., Talalay, P., and Brand, L. (1994) Biochemistry $33,7415-7422$.

31. Gryczynski, I., Steiner, R. F., and Lakowicz, J. R. (1991) Biophys. Chem. 39, 69-78.

32. Lipari, G., and Szabo, A. (1980) Biophys. J. 30, 489-506.

33. Castanho, M. A., and Prieto, M. J. (1992) Eur. J. Biochem. 207, $125-134$.

34. Chattopadhyay, A., and London, E. (1987) Biochemistry 26, 3945.

35. Kachel, K. E., Asunción-Punzalan, E., and London, E. (1995) Biochemistry 34, 15475-15479.

36. Mateo, C. R., Prieto, M., Micol, V., Shapiro, S., and Villalain, J. (2000) Biochim. Biophys. Acta 1509, 167-175.
37. Wolber, P. K., and Hudson, B. S. (1979) Biophys J. 28, 197210.

38. Castanho, M., Prieto, M., and Acuña, A. U. (1996) Biochim. Biophys. Acta 1279, 164-168.

39. de Almeida, R. F., Loura, L. M., Fedorov, A., and Prieto, M. (2002) Biophys. J. 82, 823-834.

40. Davenport, L., Dale, R. E., Bisby, R. H., and Cundall, R. B. (1985) Biochemistry 24, 4097-108.

41. Doyle, D. A, Morais Cabral, J., Pfuetzner, R. A, Kuo, A., Gulbis, J. M., Cohen, S. L., Chait, B. T., and MacKinnon, R. (1998) Science 280, 69-77.

42. Jiang, Y., Lee, A., Chen, J., Cadene, M., Chait, B. T., and MacKinnon, R. (2002) Nature 417, 515-522.

43. Backlund, B. M., and Gräslund, A. (1992) Biophys. Chem. 45, $17-25$.

44. Dietze, E. C., Wang, R. W., Lu, A. Y., and Atkins, W. M. (1996) Biochemistry 35, 6745-6753.

45. Gauduchon, P., and Wahl, P. H. (1978) Biophys. Chem. 8, 87104.

46. Laws, W. R., Ross, J. B. A., Wyssbrod, H. R., Beechem, J. M., Brand, L., and Sutherland, J. C. (1986) Biochemistry 25, 599607.

47. Cantor, C. R., and Schimmel, P. R. (1980) in Biophysical Chemistry (Barlett, A. C., Vaprek, P. C., and McCombs, L. W., Eds.) Part II, W. H. Freeman and Co., San Francisco.

48. Kinosita, K., Jr., Kawato, S., and Ikegami, A. (1977) Biophys. J. 20, 289-305

49. Kinosita, K., Jr., Ikegami, A., and Kawato, S. (1982) Biophys. J. 37, 461-464

50. Vogel, H., Nilsson, L., Rigler, R., Voges, K. P., and Jung, G. (1988) Proc. Natl. Acad. Sci. U.S.A. 85, 5067-5071.

51. Mateo, C. R., Souto, A. A., Amat-Guerri, F., and Acuna, A. U. (1996) Biophys. J. 71, 2177-2191.

52. Mateo, C. R., Lillo, M. P., Brochon, J. C., Martínez-Ripoll, M., Sanz-Aparicio, J., and Acuña, A. U. (1993) J. Phys. Chem. 97, 3486-3491.

53. Tirado, M. M., and Garcia de la Torre, J. (1979) J. Chem. Phys. $71,2581-2587$.

54. Kinosita, K., Jr., Kawato, S., and Ikegami, A. (1984) Adv. Biophys. 17, 147-203.

55. Best, L., John, E., and Jähnig, F. (1987) Eur. Biophys. J. 15, 87102.

56. Zhou, M., Morais-Cabral, J. H., Mann, S., and MacKinnon, R. (2001) Nature 411, 657-661.

$\mathrm{BI} 027183 \mathrm{H}$ 